\title{
Mapping mutations in plant genomes with the user-friendly web application CandiSNP
}

\author{
Graham J Etherington ${ }^{1,2+}$, Jacqueline Monaghan ${ }^{1 \dagger}$, Cyril Zipfel $^{1}$ and Dan MacLean ${ }^{1 *}$
}

\begin{abstract}
Background: Analysis of mutants isolated from forward-genetic screens has revealed key components of several plant signalling pathways. Mapping mutations by position, either using classical methods or whole genome high-throughput sequencing (HTS), largely relies on the analysis of genome-wide polymorphisms in $F_{2}$ recombinant populations. Combining bulk segregant analysis with HTS has accelerated the identification of causative mutations and has been widely adopted in many research programmes. A major advantage of HTS is the ability to perform bulk segregant analysis after back-crossing to the parental line rather than out-crossing to a polymorphic ecotype, which reduces genetic complexity and avoids issues with phenotype penetrance in different ecotypes. Plotting the positions of homozygous polymorphisms in a mutant genome identifies areas of low recombination and is an effective way to detect molecular linkage to a phenotype of interest.
\end{abstract}

Results: We describe the use of single nucleotide polymorphism (SNP) density plots as a mapping strategy to identify and refine chromosomal positions of causative mutations from screened plant populations. We developed a web application called CandiSNP that generates density plots from user-provided SNP data obtained from HTS. Candidate causative mutations, defined as SNPs causing non-synonymous changes in annotated coding regions are highlighted on the plots and listed in a table. We use data generated from a recent mutant screen in the model plant Arabidopsis thaliana as proof-of-concept for the validity of our tool.

Conclusions: CandiSNP is a user-friendly application that will aid in novel discoveries from forward-genetic mutant screens. It is particularly useful for analysing HTS data from bulked back-crossed mutants, which contain fewer polymorphisms than data generated from out-crosses. The web-application is freely available online at http://candisnp.tsl.ac.uk.

Keywords: High-throughput sequencing, Single nucleotide polymorphisms, Forward-genetics, Mapping, Web application

\section{Background}

Carefully designed forward-genetic screens have been an integral part of research programs for decades and remain an important tool for resolving biological pathways. Many proteins contributing to plant immune signalling have been discovered through such screens. As one example, the receptor kinase FLAGELLIN SENSING 2 (FLS2) was identified from a mutagenized Arabidopsis thaliana (hereafter, Arabidopsis) population as the receptor for bacterial flagellin [1]. The discovery of FLS2 and other surface-localized immune receptors that detect conserved

\footnotetext{
* Correspondence: dan.maclean@tsl.ac.uk

${ }^{\dagger}$ Equal contributors

'The Sainsbury Laboratory, Norwich Research Park, Norwich NR4 7UH, UK Full list of author information is available at the end of the article
}

molecular features of microbes (known as pathogenassociated molecular patterns; PAMPs) revolutionized our understanding of plant immunity [2], and reinforces the importance of genetic screens in modern research.

Genetic screens in all systems are based on similar principles. Individuals containing a phenotype of interest are first isolated from a mutagenized or naturally polymorphic population. Marker-assisted linkage analysis is then performed to identify the genomic region containing the underlying mutation(s). Finally, mutations are identified by sequence analysis and the causative mutation is usually confirmed by complementation with a non-mutated (wild-type) copy of the gene.

The most commonly used mutagenesis strategies in Arabidopsis include the induction of guanine-to-adenine 
substitutions using ethylmethane sulfonate (EMS) or the insertion of transfer-DNA using Agrobacterium tumefaciens-mediated transformation [3]. The number of mutations identified in a mapped region depends primarily on the mutagenesis. Increasing the strength of the mutagen will likely result in the recovery of more mutants containing the phenotype of interest, however this also results in more mutations in each mutant genome and can complicate correct gene identification. Mapping mutations by position classically involves out-crossing to a polymorphic ecotype and linking the phenotype of recombinant $\mathrm{F}_{2}$ individuals to molecular markers with known genomic positions, such as insertion/deletions (indels) or single nucleotide polymorphisms (SNPs). Rather than genotyping individual recombinants exhibiting the scored phenotype, linkage analysis can be performed on bulked recombinants. This process, referred to as bulk segregant analysis (BSA) [4], eases genetic analysis and is particularly effective when a large number of molecular markers are available. Although robust, these classical methods are time-consuming and labour-intensive, commonly taking more than a year (in the case of Arabidopsis) to correctly identify the causative mutation. Positional cloning can be particularly tedious in suppressor or modifier screens, where multiple loci are segregating in the mapping population. Correct identification of causative mutations depends greatly on the strength of the mutagenesis, the complexity of the cross, the penetrance of the phenotype, and the availability of molecular markers.

Recent advances in high-throughput sequencing (HTS) technologies have allowed for rapid identification of causative mutations and have been widely adopted in many fields [5]. HTS approaches have many advantages over classical mapping strategies, including a potential reduction in time and personnel needed to identify a causative mutation. Combining HTS with BSA has proven to be particularly useful [5]. Several expert operator methods utilizing HTS of bulked segregants have been described to assist plant genetic screens, including SHOREmap [6,7], Next-Generation Mapping (NGM) [8], MutMap [9-11], and others [5]. However, each of these has restrictions in their functioning that make them limited to certain types of crosses or are difficult to use for non-experts including bench-trained biologists.

The two major tools used by plant researchers start with a classical mapping approach, requiring data generated from out-crosses. SHOREmap [6,7] uses a statistic that explicitly calculates the relative abundance of alleles identified in bulked out-crossed $F_{2}$ populations and therefore relies on a priori knowledge of polymorphic allele positions in both the parental and out-crossed ecotypes. As a result, this powerful tool cannot be used when such crosses are not performed (for example, in back-crossed populations) or when genetic marker resources are not yet available. Comparatively, the marker-independent, webbased method NGM [8], does not rely on previous knowledge of ecotype-specific polymorphisms, but rather uses the ratio of the expected allele frequency of the causative mutation in bulked out-cross $F_{2}$ segregants relative to the background allele frequency of unrelated mutations. This two-step process relies first on identifying a coarse mapping interval of relative SNP paucity in which the mutation should lie; this region is usually in the order of megabases in length [8]. To further reduce the width of the coarse interval and ease identification of the causative mutation, the SNP frequency in different bands of SNP allele frequencies (overlapping groupings of similar allele frequencies; e.g., 0.5-0.6, 0.51-0.61, etc.) are compared. The point that maximises the ratio between bands representing homozygous alleles and heterozygous alleles at $50 \%$ frequency is expected to be at or around the causative mutation. The MutMap [10,11] and CloudMap [12] systems avoid the need for coarse mapping as an initial step but do not exist as user-friendly tools such that application and optimisation of parameters requires extensive expertise in a command-line computing environment.

We recently conducted a forward-genetic modifier screen in the immune-deficient bak1-5 background to identify novel components involved in plant immune signalling [13]. BRASSINOSTEROID INSENSITIVE 1ASSOCIATED KINASE 1 (BAK1) is a multi-functional co-receptor that interacts with and phosphorylates several surface-localized immune receptors including FLS2 [14-19]. Accordingly, loss-of-function bak1 alleles are strongly impaired in signalling triggered by several PAMPs [15,17-19]. We mutagenized bak1-5 seeds (in the Columbia-0 (Col-0) ecotype) with EMS and screened the $\mathrm{M}_{2}$ generation for modifier of bak1-5 (mob) mutants that restored immune signalling. To uncover the causal mob mutation(s), we back-crossed bak1-5 mob mutants to the parental line (bak1-5) and Illumina sequenced bulked $\mathrm{F}_{2}$ mob segregants. Importantly, as the parent was itself generated through EMS-mutagenesis of a transgenic Arabidopsis line $[15,20,21]$, we additionally sequenced bak1-5, which had been back-crossed for three generations prior to mutagenesis, as a reference.

We chose this approach over out-crossing to ease phenotyping and segregation analysis. First, selection of the $m o b$ mutant phenotype required scoring a quantitative response dependent on immune receptor activity, which varies in different ecotypes. For example, FLS2 in the Wassilewskija-0 (Ws-0) ecotype contains a deletion mutation resulting in a truncated and non-functional FLS2 receptor [22], while FLS2 in the Landsberg erecta-0 (Ler-0) ecotype contains polymorphisms that cause FLS2 to bind flagellin about three times stronger than FLS2 in Col-0 [23]. Second, selection of the mob mutant phenotype was dependent on bak1-5, which would not be 
present in a polymorphic ecotype such as Ler-0 and would thus need to be genotyped prior to phenotype scoring. Similar considerations would likely arise in any screen involving second-site modifier or suppressor mutations.

While a back-cross simplifies genetic analysis, bulk segregant sequence analysis is complicated by far fewer segregating SNPs (1 SNP every 65,000 bp) compared to out-crosses (1 SNP every $900 \mathrm{bp}$ ) (Additional file 1). Although few in number, we found that simply plotting the position of SNPs with close-to-homozygous alternate allele frequencies along the chromosomes was a convenient and easy way of performing bulk segregant linkage analysis from a back-crossed population. We developed this method into a user-friendly web-based application, called CandiSNP, which generates density plots from SNP data obtained from HTS. We demonstrate the utility of CandiSNP by analysing sequence data generated from two allelic mob mutants, bak1-5 mob1 and bak1-5 mob2, which are caused by mutations in the gene encoding the calcium-dependent protein kinase CPK28 [13].

\section{Implementation}

CandiSNP is part of a straightforward and flexible workflow

To provide a publicly available easy-to-use tool for mapping mutations, we developed the CandiSNP web application (Figure 1). Prior to using CandiSNP, users must identify SNP positions. Typically this would be done in a workflow that maps quality-controlled (QC) reads to the appropriate reference genome and provides SNP position data as input (Figure 2A). The data must be provided in a simple comma-delimited format and must have the following column headers: 'Chr', 'Pos', 'Ref', 'Alt' and

\section{CandiSNP: identifying candidate SNPs in genomes

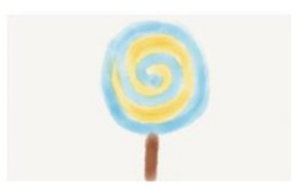 \\ version 0.2 .0 - "beau jour blaireau" \\ CandiSNP analyses and provides per-chromosome plots of SNPs across genomes. It provides the location of the SNPs and uses colour-coded palettes to show if the SNPs are in coding or non-coding regions and if they are synonymous or non- synonymous.}

\section{Reset data fields}

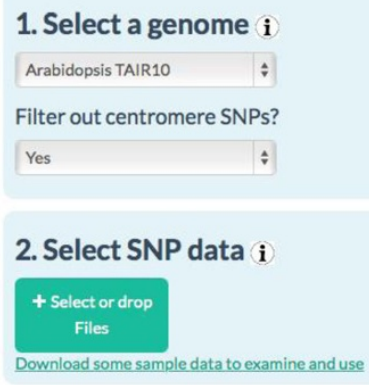

3. Select palette to view SNPs i

CandisNP $\quad$;

4. Upload and analyse

OAnalyse See the outputhelpguide

Figure 1 Screen-shot of the CandiSNP web application. CandiSNP is openly accessible online at http://candisnp.tsl.ac.uk. The application is laid out so users can make their way through the application in numbered steps. Users choose which genome they would like to use for comparison (the program currently supports Arabidopsis, rice, tomato, grape, maize and soybean genomes). The option of filtering SNPs concentrated around centromeres is also provided. Users then upload their SNP data file, indicate their preferred allele frequency cut-off, and choose from a number of different palettes for SNP visualization. 


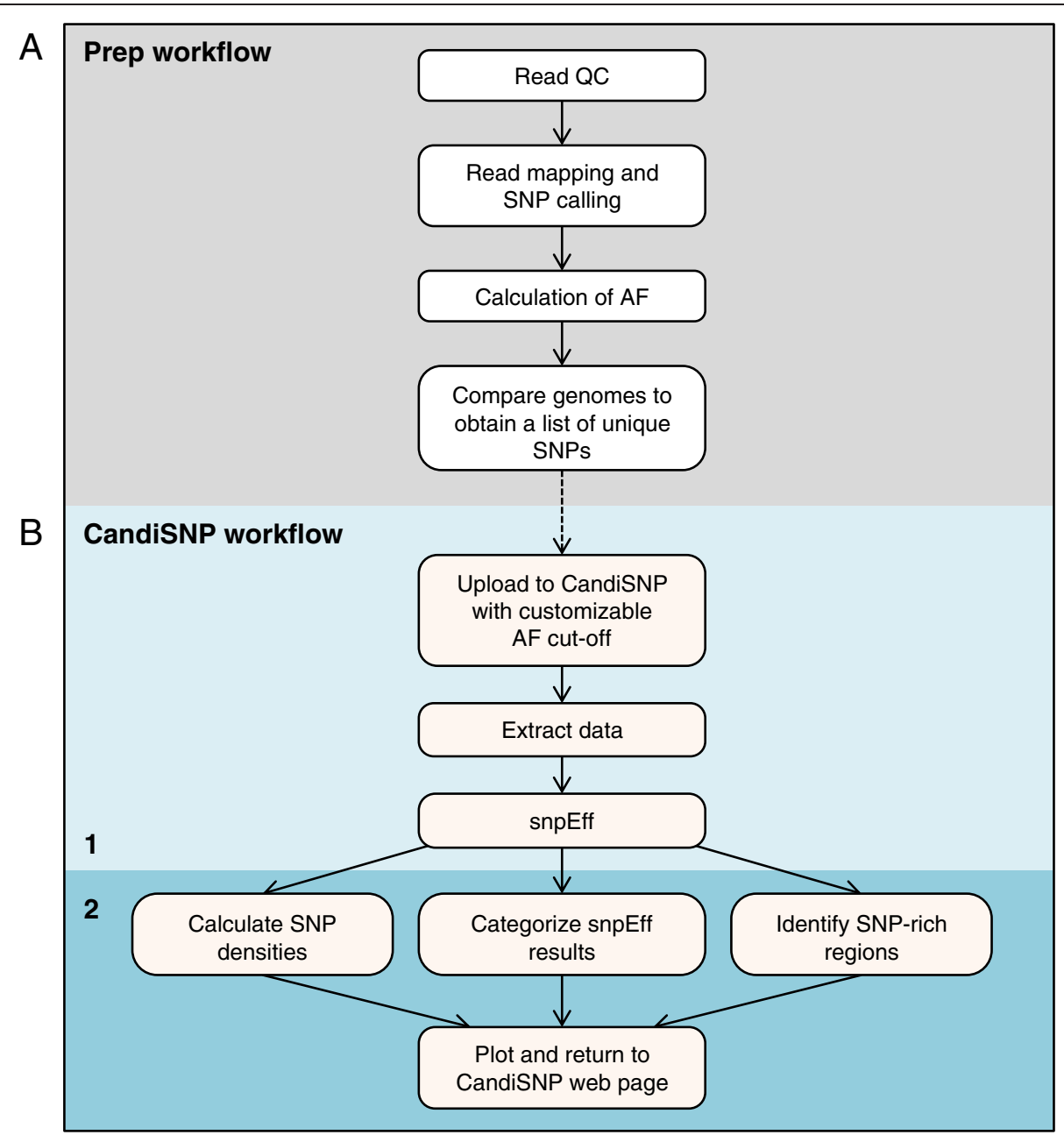

Figure 2 Bioinformatics pipeline for sequence analysis. Pipeline indicating the preparatory steps required by the user (A) prior to running the CandiSNP web application (B).

'Allele_Freq' (meaning: Chromosome, Position, Reference base, Alternate base, and Allele Frequency, respectively). To create CandiSNP input files, we suggest using the pileups_to_snps.rb Ruby script (Additional file 2 and https://github.com/danmaclean/candisnp/blob/master/ pileup_to_snps.rb). By using this generic and flexible input format, our system allows the user to take advantage of existing pipelines and data, including files generated by external service providers or even datasets originating from other technologies.

CandiSNP analysis is a two-step process (Figure 2B). CandiSNP first uses snpEff [24] to categorise SNPs according to their position in genomic features. The SNP predictions are categorised as: (1) Causing a change in an intergenic (non-annotated) region, (2) Causing a synonymous change in an annotated protein-coding region, or (3) Causing a non-synonymous change in an annotated protein-coding region. CandiSNP then creates a chromosome map visualizing the position of all SNPs meeting a user-selected (and customizable) alternate allele frequency (AF) threshold, and renders this information by colouring SNPs according to category. SNPs in category (3) are represented in a colour that highlights their priority as putative causative SNPs. The density and distribution of SNPs is also visualized as a line graph below each chromosome. The user is provided with a downloadable list of genomic effects for all inputted SNPs and is provided with a Scalable Vector Graphic figure of publication quality that can be easily exported.

Currently, CandiSNP supports several plant genomes including Arabidopsis thaliana Col-0 TAIR9 and TAIR10 [25], Oryzae sativa v7 [26], Solanum lycopersicum v2.40 [27], Glycine max 1.09v8 [28], Vitus vinefera v1 [29] and Zea mays $\mathrm{B} 73 \mathrm{v} 5 \mathrm{~b}$ [30].

\section{Design and availability}

CandiSNP is available in multiple formats for users with diverse security and confidentiality needs and differing 
access to computational infrastructure. Primarily, CandiSNP is provided as a web application, available at http://candisnp.tsl.ac.uk. The web application takes text files as input. Instructions are provided on-screen to assist users new to the tool. The web application requires no registration and does not collect user information. For laboratories with bioinformatics support wishing to use an internal and private version of the web application, we provide a package and source code in Perl/HTML/Javascript for free download and use under the GNU GPL3 Licence, from the dedicated code hosting website GitHub at https://github.com/danmaclean/candisnp. For those wishing to run the CandiSNP process on a command line as part of bioinformatics pipelines, a Perl module is also available as part of the source code.

\section{Results and discussion Case study}

Bulk segregant analysis of two mob mutants using Illumina sequencing identifies thousands of polymorphisms

As a case study for CandiSNP we examined HTS data obtained for two allelic recessive mutants, bak1-5 mob1 and bak1-5 mob2, that were isolated from the modifier of bak1-5 (mob) screen [13]. Both bak1-5 mob1 and bak1-5 mob2 were back-crossed with bak1-5 and the $\mathrm{F}_{2}$ populations were screened for the mob phenotype (Figure 3A). $\mathrm{F}_{2}$ segregants that displayed the mob phenotype were bulked and genomic DNA was isolated. The bak1-5 parental genome was prepared by harvesting individuals from a homozygous back-crossed line. DNA samples were sent to the Beijing Genomics Institute (BGI, Hong Kong) for library construction and 90 bp paired-end sequencing on the Illumina HiSeq platform.

With the aid of FASTQC v 0.10.1 [31] in the Galaxy platform [32-34], all reads were quality controlled (QC) so that reads that contained undefined nucleotides, were not $90 \mathrm{bp}$ long, or were full-length homopolymer runs were removed. Reads containing nucleotides with a Sangerscaled PHRED quality score of less than 10 at the $3^{\prime}$ end were trimmed to this minimum using Sickle version 1.21.0 [35]. The QC pipeline is available as a Galaxy workflow at http://dx.doi.org/10.6084/m9.figshare.1248898.

$\mathrm{QC}$ reads were then mapped to the TAIR10 genome [25] using the BWA v 0.6.1 aligner [36]. For the bak1-5 genome, we aligned 47.9 million 90 bp paired-end reads, with a mean insert size of $467 \mathrm{bp}$, to the TAIR10 Arabidopsis reference sequence ( $98.5 \%$ of reads aligned). We similarly aligned 47.2 million 90 bp paired-end reads, with a mean insert size of $452 \mathrm{bp}$, to TAIR10 for the bak15 mob2 genome (99.6\% of reads aligned). Average alignment depth over the nuclear chromosomes was 36 for bak1-5 and 59.3 for bak1-5 mob2. Details regarding the bak1-5 mob1 genome sequence have been previously described [13].
After alignment, SNPs were identified and allele frequencies were calculated using SAMtools v 0.1.8 [37]. Reads with mapping quality scores less than 20 and individual bases with sequence quality less than 20 were discarded. Genome positions where the reference base was unknown were excluded. Positions were considered SNPs if they had a minimum read coverage of 6 and a maximum of 250. The alignment and SNP calling workflow is available at http://dx.doi.org/10.6084/m9.figshare. 1171109. In total, bak1-5 contained 2,639 SNPs compared to Col-0, while bak1-5 mob1 and bak1-5 mob2 contained 4,188 and 3,581 SNPs, respectively (Table 1).

\section{Filtering of non-unique SNPs in the mutants reduces complexity}

To reduce the complexity of the bak1-5 mob1 and bak15 mob2 datasets, we compared SNP calls from the different genome sequences and removed SNPs that either mob had in common with each-other or bak1-5. Comparing the bak1-5 mob genomes to the parental bak1-5 genome identified 2,111 and 2,132 SNPs shared between bak1-5 and either bak1-5 mob1 or bak1-5 mob2, respectively (Figure 3B). There were an additional 444 SNPs that were shared between the bak1-5 mob1 and bak1-5 mob2 genomes that were not identified in bak15 . We reasoned that these shared SNPs were contributed by bak1-5 but were not identified due to low sequence coverage in those areas. These analyses identified 2,746 polymorphisms that were shared between at least two of the genomes. Discarding these allowed us to identify over 1,000 SNPs that were uniquely present in bak1-5 mob1 and bak1-5 mob2 (Figure 3B). The general value of comparing multiple mutant sequences to remove shared SNPs has been previously demonstrated [12] and the general case discussed in Additional file 3. However, deleting all common SNPs in this way precludes identification of identical causative SNPs in different mutants, which, while extremely rare, is something to consider prior to performing such analysis.

\section{CandiSNP enables easy visual assessment of SNP positions and finds genomic regions with low recombination linked to the phenotype of interest}

Positional cloning is based on linking phenotypes to molecular markers with known genomic positions. If recessive, $F_{2}$ recombinants that contain the phenotype of interest are homozygous for the unknown mutation. Identifying molecular markers that are invariably homozygous for the mutant type are therefore linked to the scored phenotype and thus the mutation. As we conducted a back-cross rather than an out-cross, the only molecular markers we could use were those resulting from the comparative analysis just described between the parental and mutant genomes. 


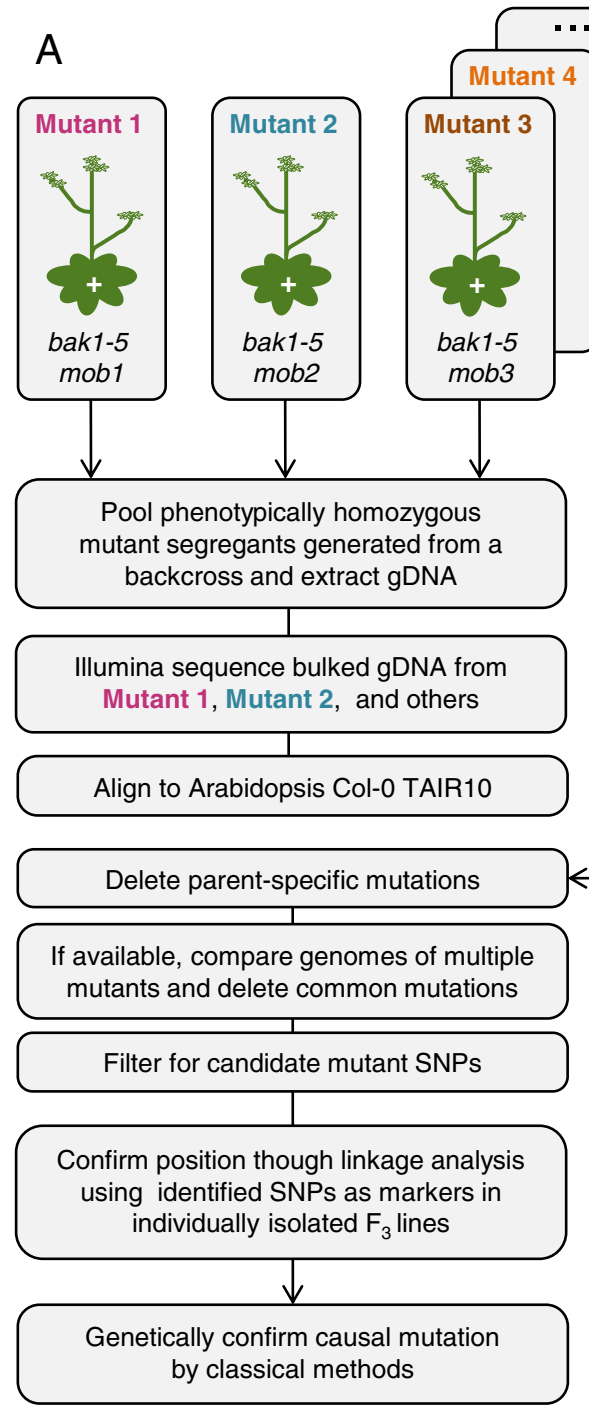

B

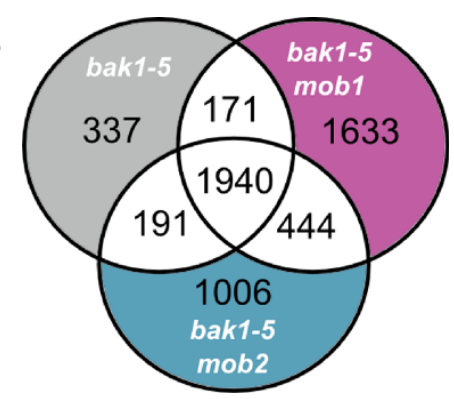

Figure 3 Pipeline for bulking segregants and identification of unique SNPs. (A) The recessive bak1-5 mob1 and bak1-5 mob2 mutants were back-crossed to the parent bak1-5, allowed to self-fertilize in the $F_{1}$, and phenotypically scored in the $F_{2}$ for the mob phenotype. Positive segregants were bulk harvested and genomic DNA was prepared and sequenced using the Illumina HiSeq platform. For comparison, the bak $1-5$ genome was also sequenced. A similar genetics pipeline could be employed for dominant mutants, but material would need to be bulked from segregants that were phenotypically verified as homozygous in the $\mathrm{F}_{3}$ generation. (B) A three-way comparison between the bak1-5, bak1-5 mob1, and bak1-5 mob2 genomes identified the total number of unique SNPs in each genome.

To identify which of the over 1,000 unique SNPs in the mutant genomes are linked to the scored phenotype, it is necessary to determine which SNPs are homozygous or close-to-homozygous. Although bulking mutants increases the likelihood of identifying homozygous SNPs (in theory, with an allele frequency of 100\%), some margin of error must be allowed to account for sequencing and phenotyping errors. CandiSNP facilitates the easy discovery of a useful frequency cut-off by allowing the user to iteratively refine the allele frequency and view a new plot concurrent with previous ones for comparison. As a further refinement of the CandiSNP web application, we included the option of removing SNPs concentrated around centromeres (in organisms where a centromere is defined in the genome assembly), as these are areas of low recombination frequency and tend to skew density analysis. After selecting an allele frequency threshold, CandiSNP plots the positions of retained SNPs as dots across the chromosomes and highlights SNPs of different classes according to a selected palette. The per-chromosome density and distribution of SNPs is rendered in a second plot to aid in cases with high numbers of SNPs. In our case study we chose $75 \%$ as an acceptable frequency cutoff, and used CandiSNP to identify 88 and 143 unique 
Table 1 Identification of unique and candidate SNPs in the parental and mutant genomes

\begin{tabular}{|c|c|c|c|}
\hline & bak1-5 & bak1-5 mob1 & bak1-5 mob2 \\
\hline $\begin{array}{l}\text { Total SNPs compared to } \\
\text { Col-0 TAIR10 }\end{array}$ & 2639 & 4188 & 3581 \\
\hline $\begin{array}{l}\text { Unique SNPs compared } \\
\text { to the parent }\end{array}$ & 2639 & 1633 & 1006 \\
\hline Unique SNPs, AF >75\% & 785 & 88 & 143 \\
\hline $\begin{array}{l}\text { Unique SNPs, AF }>75 \% \text {, } \\
\text { annotated coding }\end{array}$ & 240 & 16 & 41 \\
\hline $\begin{array}{l}\text { Unique SNPs, AF }>75 \% \text {, } \\
\text { annotated coding, non-synon }\end{array}$ & 168 & $9^{a}$ & $31^{\mathrm{b}}$ \\
\hline
\end{tabular}

To identify SNPs unique to each genome, the parental and mutant genomes were compared and filtered. Unique SNPs in bak1-5 refer to those that are not found in the Col-0 TAIR10 genome. For the bak1-5 mob1 and bak1-5 mob2 datasets, SNPs shared between any of the three genomes (bak1-5, bak1-5 mob1 and bak1-5 mob2; Figure 3B) were removed, resulting in SNPs uniquely found in each of those genomes. Filtering for SNPs with an allele frequency higher than 75\% that cause non-synonymous ('non-synon') changes in annotated coding regions resulted in a list of candidate causative mutations.

${ }^{a}$ Candidate causative SNPs for bak1-5 mob1 are listed in Table 2.

${ }^{b}$ Candidate causative SNPs for bak1-5 mob2 are listed in Table 3.

SNPs meeting this requirement in the bak1-5 mob1 (Additional file 4) and bak1-5 mob2 (Additional file 5) genomes, respectively (Table 1 ). CandiSNP further identified 9 and 31 candidate causative SNPs (those which cause non-synonymous changes in protein-coding regions) for bak1-5 mob1 (Table 2; Additional file 4) and bak1-5 mob2 (Table 3; Additional file 5). By choosing a palette to highlight candidate SNPs (shown in our case study as red dots) we observe putative map positions for both mutants at the bottom of chromosome 5 (Figure 4).

Therefore, CandiSNP visualizes the location of SNPs linked to a phenotype of interest. Moreover, CandiSNP provides annotations describing the genomic feature in which each SNP is located. This function provides useful information for biologists who can make conceptual links between biological knowledge and molecular function of the genomic feature and refine candidate lists further. On its own this annotation function provides a fast and easy way of finding the effect of any mutation in the supported genomes, enhancing the usefulness of CandiSNP beyond that of mutant mapping.

\section{Fine mapping and confirmation of causative SNPS}

We confirmed the presence of candidate causative SNPs by Sanger sequencing polymerase chain reaction (PCR)generated amplicons containing the predicted mutations prepared from individual back-crossed $\mathrm{F}_{3}$ bak1-5 mob1 and bak1-5 mob2 plants compared to the bak1-5 parent (Tables 2 and 3). Using these SNPs as molecular markers allowed us to further map the mutations by position and narrowed the list of candidate causative SNPs down to 3 in bak1-5 mob1 and 6 in bak1-5 mob2. Primers used for this analysis are available in Additional file 6. Alternative methods for such analysis could include other allelespecific genotyping methods such as designing cleaved amplified polymorphic sequence (CAPS) markers [38] or conducting high-resolution melting (HRM) analysis [39] on PCR amplicons. We previously reported genetic confirmation that the polymorphic CPK28 alleles contained within these lists of candidate SNPs were causative of the bak1-5 mob1 and bak1-5 mob2 mutant phenotypes [13]. Our analysis was simplified by knowledge of allelism between the two mutants, which clearly indicated CPK28 as the causative locus. In the absence of such knowledge, marker-assisted genotyping of additional homozygous $\mathrm{F}_{3}$ lines could further reduce the number of candidate mutations and ease genetic confirmation.

\section{CandiSNP performance}

As CandiSNP is a predictive classification method for determining whether a given SNP is a causative mutation, it is important that we estimate the accuracy of the

Table 2 Candidate causative SNPs in bak1-5 mob1

\begin{tabular}{|c|c|c|c|c|c|c|c|}
\hline Chr & Position & Ref/Alt & AF (\%) & AGI & Gene ID & AA change & Sanger $F_{3}$ \\
\hline 1 & 11892068 & $\mathrm{C} / \mathrm{T}$ & 100 & At1g32830 & Transposable element & n.a. & Absent \\
\hline 1 & 11892070 & $\mathrm{G} / \mathrm{T}$ & 100 & At1g32830 & Transposable element & n.a. & $\mathrm{Hom}^{\mathrm{a}}$ \\
\hline 1 & 11892252 & $\mathrm{~T} / \mathrm{G}$ & 100 & At1g32830 & Transposable element & n.a. & $\mathrm{Hom}^{\mathrm{a}}$ \\
\hline 1 & 16516501 & $\mathrm{~T} / \mathrm{C}$ & 83.3 & At1g43745 & Transposable element & n.a. & Absent \\
\hline 1 & 16525522 & $\mathrm{~T} / \mathrm{C}$ & 77.8 & At1g43755 & Transposable element & n.a. & $\mathrm{Hom}^{\mathrm{a}}$ \\
\hline 1 & 24243231 & $\mathrm{G} / \mathrm{A}$ & 80.9 & At1g65270 & Unknown protein & $G>S$ & Hom \\
\hline 5 & 26457834 & $\mathrm{G} / \mathrm{A}$ & 85.0 & At5g66210 & CPK28 & $A>V$ & $\mathrm{Hom}^{\mathrm{b}}$ \\
\hline 5 & 26458077 & $\mathrm{G} / \mathrm{A}$ & 78.5 & At5g66210 & CPK28 & $S>L$ & $\mathrm{Hom}^{\mathrm{b}}$ \\
\hline 5 & 26474069 & $\mathrm{G} / \mathrm{A}$ & 76.5 & At5g66270 & Zn-finger family protein & $P>L$ & Hom \\
\hline
\end{tabular}

Unique SNPs in annotated coding regions with allele frequencies (AF) over 75\% identified by CandiSNP for bak1-5 mob1, listing the Chromosome number (Chr), position, reference base (Ref), sequenced alternate base (Alt), locus number (AGI), gene identification (Gene ID), amino acid change (AA change; 'n.a.' is not applicable). All SNPs were confirmed in at least three independent back-crossed lines ( $F_{3}$ generation) by Sanger sequencing compared to bak $1-5$. SNPs that were homozygous (Hom) or not present (Absent) are listed.

${ }^{a}$ These SNPs were also identified in bak1-5 by Sanger sequencing (however, not by lllumina sequencing) and are therefore not unique to bak1-5 mob1.

${ }^{\mathrm{b}}$ These SNPs are the causative mutations for bak1-5 mob1 [13]. 
Table 3 Candidate causative SNPs in bak1-5 mob2

\begin{tabular}{|c|c|c|c|c|c|c|c|}
\hline Chr & Position & Ref/ Alt & AF (\%) & AGI & Gene ID & AA change & Sanger $F_{3}$ \\
\hline 1 & 7446564 & $\mathrm{C} / \mathrm{T}$ & 76.2 & At1g21270 & WAK2 & $P>L$ & Seg \\
\hline 1 & 11892984 & $\mathrm{C} / \mathrm{T}$ & 100 & At1g32830 & Transposable element & n.a. & $\mathrm{Hom}^{\mathrm{a}, \mathrm{b}}$ \\
\hline 1 & 16513961 & $\mathrm{~T} / \mathrm{G}$ & 77.1 & At1g43740 & Transposable element & n.a. & $\mathrm{Hom}^{\mathrm{a}, \mathrm{b}}$ \\
\hline 1 & 17757465 & $\mathrm{C} / \mathrm{T}$ & 76.0 & At1g48090 & Calcium-dependent lipid-binding protein & $D>N$ & Seg \\
\hline 1 & 18192647 & $\mathrm{C} / \mathrm{T}$ & 76.0 & At1g49190 & ARR19 & $R>W$ & Seg \\
\hline 1 & 22178447 & $\mathrm{C} / \mathrm{T}$ & 82.6 & At1g60140 & TPS10 & $\mathrm{D}>\mathrm{N}$ & Hom \\
\hline 2 & 2568811 & $\mathrm{C} / \mathrm{T}$ & 83.3 & At2g06470 & Transposable element & n.a. & $\mathrm{Hom}^{\mathrm{a}}$ \\
\hline 2 & 5277241 & $\mathrm{~T} / \mathrm{G}$ & 100 & At2g12850 & Transposable element & n.a. & Not tested \\
\hline 4 & 2362567 & $\mathrm{C} / \mathrm{A}$ & 100 & At4g04655 & Transposable element & n.a. & $\mathrm{Seg}^{\mathrm{a}}$ \\
\hline 5 & 5569896 & $\mathrm{G} / \mathrm{A}$ & 94.1 & At5g16930 & AAA-type ATPase family protein & W > stop & Seg \\
\hline 5 & 14285189 & $\mathrm{G} / \mathrm{A}$ & 81.3 & At5g36260 & Eukaryotic aspartyl protease family protein & $S>L$ & Absent \\
\hline 5 & 14579245 & $\mathrm{G} / \mathrm{A}$ & 75.0 & At5g36935 & Transposable element & n.a. & Not tested \\
\hline 5 & 15751875 & $\mathrm{G} / \mathrm{A}$ & 77.2 & At5g39350 & Tetratricopeptide repeat-like superfamily protein & $R>K$ & Seg \\
\hline 5 & 17503318 & $\mathrm{G} / \mathrm{A}$ & 82.7 & At5g43560 & TRAF-like superfamily protein & $E>K$ & Seg \\
\hline 5 & 17597830 & $\mathrm{G} / \mathrm{A}$ & 80.0 & At5g43800 & Transposable element & n.a. & Not tested \\
\hline 5 & 17820568 & $\mathrm{G} / \mathrm{A}$ & 93.3 & At5g44240 & ALA2 & $A>T$ & Seg \\
\hline 5 & 18251689 & $\mathrm{G} / \mathrm{A}$ & 83.3 & At5g45140 & Nuclear RNAP2 & $P>S$ & Seg \\
\hline 5 & 18261108 & $\mathrm{G} / \mathrm{A}$ & 75.0 & At5g45150 & RTL3 & $D>N$ & Seg \\
\hline 5 & 18399206 & $\mathrm{G} / \mathrm{A}$ & 80.9 & At5g45400 & RPA70C & $V>M$ & Seg \\
\hline 5 & 21859555 & $\mathrm{G} / \mathrm{A}$ & 90.0 & At5g53840 & F-box/RNI-like/FBD-like domains-containing protein & $S>F$ & Seg \\
\hline 5 & 21939106 & $\mathrm{G} / \mathrm{A}$ & 80.9 & At5g54062 & Unknown protein & $E>K$ & Seg \\
\hline 5 & 22002355 & $\mathrm{G} / \mathrm{A}$ & 88.9 & At5g54203 & Transposable element & n.a. & Absent \\
\hline 5 & 22066915 & $\mathrm{G} / \mathrm{A}$ & 79.3 & At5g54340 & $\mathrm{C} 2 \mathrm{H} 2$ and $\mathrm{C} 2 \mathrm{HC}$ zinc-finger superfamily protein & $V>1$ & Seg \\
\hline 5 & 22430866 & $\mathrm{G} / \mathrm{A}$ & 90.0 & At5g55310 & TOP1 $\beta$ & $A>V$ & Seg \\
\hline 5 & 22565056 & $\mathrm{G} / \mathrm{A}$ & 76.5 & At5g55750 & Hydroxyproline-rich glycoprotein family protein & $P>S$ & Seg \\
\hline 5 & 26458017 & $\mathrm{C} / \mathrm{T}$ & 93.1 & At5g66210 & CPK28 & $W>$ stop & $\operatorname{Hom}^{c}$ \\
\hline 5 & 26560691 & $\mathrm{C} / \mathrm{T}$ & 95.8 & At5g66550 & Maf-like protein & $G>R$ & Hom \\
\hline 5 & 26626055 & $\mathrm{C} / \mathrm{T}$ & 78.9 & At5g66690 & UGT72E2 & $P>S$ & Hom \\
\hline 5 & 26710709 & $\mathrm{C} / \mathrm{T}$ & 75.0 & At5g66880 & SNRK2.3 & $P>S$ & Hom \\
\hline 5 & 26716839 & $\mathrm{C} / \mathrm{T}$ & 80.9 & At5g66900 & CC-NB-LRR family protein & $D>N$ & Hom \\
\hline 5 & 26935248 & $\mathrm{C} / \mathrm{T}$ & 75.0 & At5g67500 & VDAC2 & $T>1$ & Hom \\
\hline
\end{tabular}

Unique SNPs in annotated coding regions with allele frequencies (AF) over 75\% identified by CandiSNP for bak1-5 mob2, listing the Chromosome number (Chr), position, reference base (Ref), sequenced alternate base (Alt), locus number (AGI), gene identification (Gene ID), amino acid change (AA change; 'n.a.' is not applicable). All SNPs were confirmed in at least three independent back-crossed lines ( $F_{3}$ generation) by Sanger sequencing compared to bak $1-5$. SNPs that were homozygous (Hom), segregating (Seg), not identified (Absent), or not tested are listed.

aThese SNPs were also identified in bak1-5 by Sanger sequencing (however, not by lllumina sequencing) and are therefore not unique to bak1-5 mob2.

${ }^{\mathrm{b}}$ These are single base deletion mutations.

'This SNP is the causative mutation for bak1-5 mob2 [13].

classifications made. One useful approach for assessing the overall accuracy of the analysis, rather than each individual prediction, post hoc, is to construct a receiver operating characteristic (ROC) curve [40]. In such an analysis, a set of independently verified 'true positive' results are compiled and the ability of the classifier to recall these at different parameters is plotted. To assess CandiSNP we used the bak1-5 mob1 and bak1-5 mob2 verified causative SNPs and varied the allele frequency parameter to carry out a standard ROC analysis [40] (Additional file 7). True positives were defined as verified causative SNPs and false positives were defined as any non-causative SNP identified by CandiSNP regardless of location and category. False negatives were defined as causative SNPs not included in that threshold and true negatives as any position in the genome where a SNP was not identified (i.e., genome size - false positives). Sensitivity was calculated as the number of true positives divided 


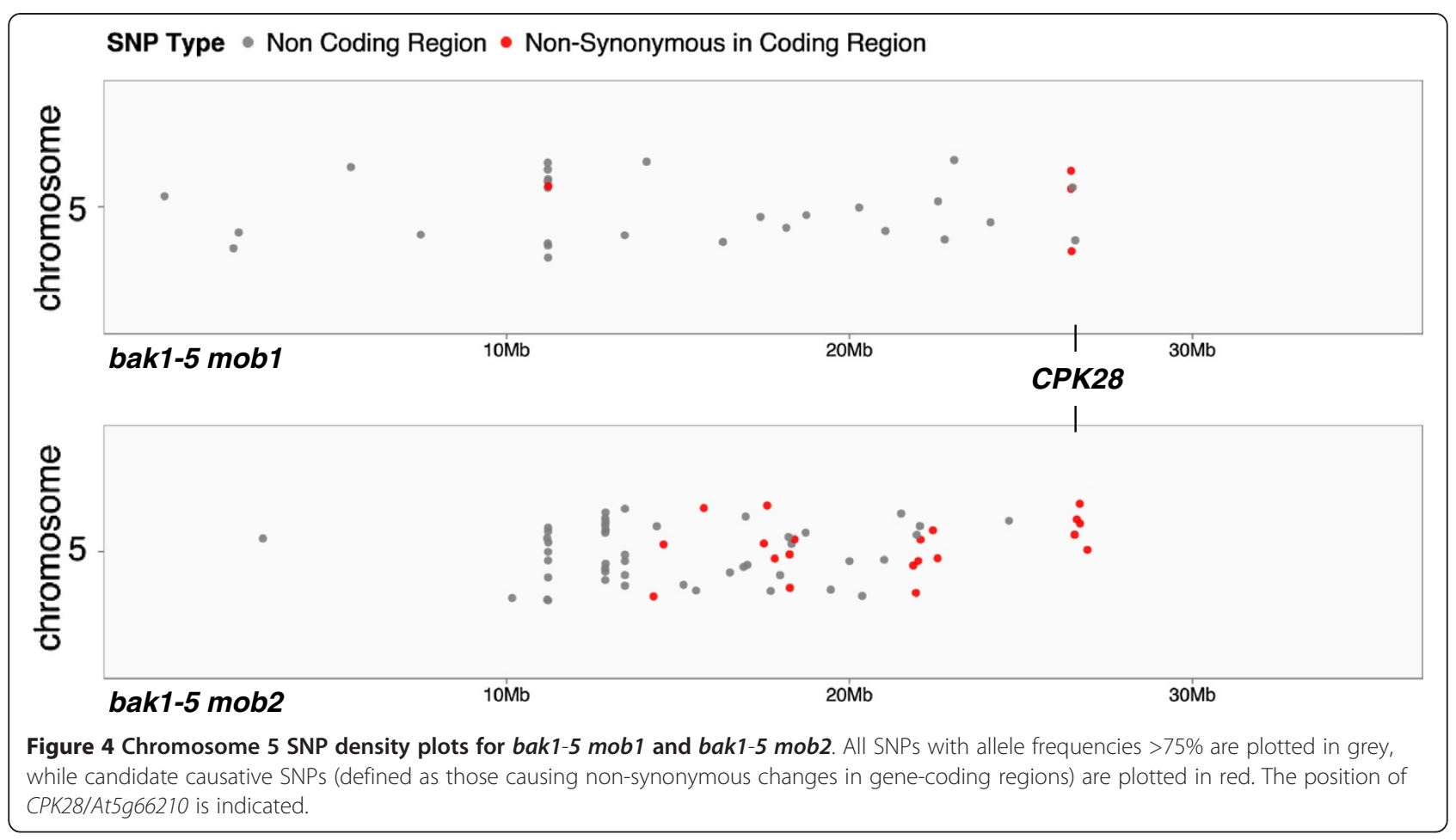

by the total of true positives and false negatives. Specificity was calculated as the number of true negatives divided by the number of false positives and true negatives. Sensitivity assesses the ability of CandiSNP to recall the verified SNPs whilst specificity assesses the ability of CandiSNP to exclude non-causative SNPs.

For our test case, the sensitivity of CandiSNP drops completely for allele frequencies over $75 \%$ (Additional file 7), indicating that accounting for phenotype penetrance and sequencing errors is an important factor in the pipeline. Further, rather than reducing the number of errors, setting an overly stringent allele frequency causes the pipeline to fail by screening out real candidates. Specificity remains high across all possible allele frequencies, mostly due to the masking effect of a very high true negative count. The closely related false positive score shows a decrease to less than $25 \%$ of the candidate SNP list after an allele frequency of $62 \%$. While the absolute optimum for our data is at $75 \%$ (i.e., the maximisation of sensitivity and minimisation of false positives), taken as a whole the ROC analysis indicates that allele frequencies of $60 \%$ to $75 \%$ represent a likely 'best trade-off' window for CandiSNP analysis.

\section{Conclusions}

Genetic screens have revealed important regulators of signal transduction pathways and remain an important tool in modern research. Although greatly accelerated with the advent of HTS technologies, correct identification of causative mutation(s) remains a bottleneck in forward-genetics. To increase the repertoire of programs available to plant geneticists, we developed the CandiSNP web application, which is particularly useful for datasets containing few SNPs. In our test case, CandiSNP successfully identified causative SNPs in two recessive mutants after bulking phenotypically homozygous $F_{2}$ segregants generated from a back-cross. We propose that CandiSNP could additionally be used to identify causative SNPs in dominant mutants as long as they are verified to be phenotypically homozygous in the $F_{3}$ prior to bulk segregant analysis. By plotting homozygous and close-tohomozygous SNPs identified from HTS along the chromosome arms, the program visualizes areas of linkage and easily narrows down candidate mutation positions. CandiSNP is both fast and accurate, producing highquality editable graphics in a matter of minutes. CandiSNP is a user-friendly web application that will facilitate gene discovery in plant genetic screens.

\section{Availability requirements}

Project name: CandiSNP.

Project home page: http://candisnp.tsl.ac.uk

(Source code is available under the GPLv3 open-source license at https://github.com/danmaclean/candisnp).

Operating system(s): All systems capable of running a modern web-browser.

Other requirements: Internet connection.

License: GPL3 (http://www.gnu.org/licenses/licenses.html).

Any restrictions: None. 


\section{Additional files}

Additional file 1: Calculation of expected SNP frequencies in out- and back-crosses. SNP frequencies are calculated from different types of crosses using available data.

Additional file 2: SNP calling script. To create our CandiSNP input files we used the pileups_to_snps.rb Ruby script which relies on the bio-samtools and bio-gngm Ruby Gems. The input data is the FASTA reference sequence (TAIR10 genome in this case) and the SAM/BAM alignment file for each dataset. The output is a comma-delimited file of SNPs formatted for input to the CandiSNP application. The source code for this script is available at https://github.com/danmaclean/candisnp/ blob/master/pileup_to_snps.rb.

Additional file 3: Simplifying SNP analysis by comparing multiple mutant genomes. Comparing multiple mutant genomes identifies unique SNPs in each mutant

Additional file 4: Genome-wide SNP density plots for bak1-5 mob1. Using the 'CandiSNP palette', all SNPs with allele frequencies $>75 \%$ are plotted in grey, while candidate causative SNPs (defined as those causing non-synonymous changes in gene-coding regions) are plotted in red. These plots are also available at http://dx.doi.org/10.6084/m9.figshare.1250028.

Additional file 5: Genome-wide SNP density plots for bak1-5 mob2. Using the 'CandiSNP palette', all SNPs with allele frequencies $>75 \%$ are plotted in grey, while candidate causative SNPs (defined as those causing non-synonymous changes in gene-coding regions) are plotted in red. These plots are also available at http://dx.doi.org/10.6084/m9.figshare.1250027.

Additional file 6: Primers used in this study. Genomic regions containing candidate SNPs in bak1-5 mob1 and bak1-5 mob2 were amplified by PCR using these primers and Sanger-sequenced alongside bak1-5 as a control.

Additional file 7: Receiver operating characteristic curve plot. Receiver operating characteristic curve plot, demonstrating the optimal parameters for use in CandiSNP on bak1-5 mob1. The proportion of false positives (non-causative SNPs identified by CandiSNP regardless of location and category) is also plotted as a comparison to the standard sensitivity and specificity [40]. The overlaid dashed line represents the optimal point that maximises the ability of CandiSNP to find real causative SNPs (sensitivity) while minimising the inclusion of false positives.

\section{Abbreviations}

AF: Allele frequency; Alt: Alternative sequence; BAK1: BRASSINOSTEROID INSENSITIVE 1-ASSOCIATED KINASE 1; Bp: base pair; BWA: Burrows-Wheeler aligner; CAPS: Cleaved amplified polymorphic sequence; CandiSNP: Candidate SNP; Chr: Chromosome; CPK28: CALCIUM-DEPENDENT PROTEIN KINASE 28; Col-0: Columbia-0; EMS: Ethylmethane sulfonate; F: Filial generation; FLS2: FLAGELLIN SENSING 2; HRM: High-resolution melting; Indel: Insertion/ deletion; Ler-0: Landsberg erecta-0; M: Mutant generation; Mob: modifier of bak1-5; NGM: Next-generation mapping; HTS: High-throughput sequencing; PCR: Polymerase chain reaction; Pos: Position; SAM: Sequence alignment/map; SNP: Single nucleotide polymorphism; TAIR: The Arabidopsis Information Resource; QC: Quality control; Ws-0: Wassilewskija-0.

\section{Competing interests}

The authors declare that they have no competing interests.

\section{Authors' contributions}

JM performed the mutant screen and bulk segregant analysis and worked with GE and DM for data analysis and program development under the supervision of CZ. GE and DM performed bioinformatics and created the CandiSNP web application. All authors contributed to writing the paper. All authors read and approved the final manuscript.

\section{Acknowledgements}

We thank Dr. Ghanasyam Rallapalli and Dr. Frederikke Gro Malinovsky for careful reading of the manuscript and all members of the MacLean and Zipfel laboratories for helpful suggestions. Martin Page is acknowledged for technical assistance. This research was funded by grants from The Gatsby Foundation
(DM, CZ) and The European Research Council (CZ). JM was the recipient of a Long-Term Fellowship from the European Molecular Biology Organization.

\section{Author details}

${ }^{1}$ The Sainsbury Laboratory, Norwich Research Park, Norwich NR4 7UH, UK. ${ }^{2}$ The Genome Analysis Centre, Norwich Research Park, Norwich NR4 7UH, UK.

Received: 22 October 2014 Accepted: 5 December 2014

Published online: 30 December 2014

\section{References}

1. Gomez-Gomez L, Boller T: FLS2: an LRR receptor-like kinase involved in the perception of the bacterial elicitor flagellin in Arabidopsis. Mol Cell 2000, 5(6):1003-1011.

2. Boller T, Felix G: A renaissance of elicitors: perception of microbe-associated molecular patterns and danger signals by pattern-recognition receptors. Annu Rev Plant Biol 2009, 60:379-406.

3. Page DR, Grossniklaus U: The art and design of genetic screens: Arabidopsis thaliana. Nat Rev Genet 2002, 3(2):124-136.

4. Michelmore RW, Paran I, Kesseli RV: Identification of markers linked to disease-resistance genes by bulked segregant analysis: a rapid method to detect markers in specific genomic regions by using segregating populations. Proc Natl Acad Sci U S A 1991, 88(21):9828-9832.

5. Schneeberger $K$ : Using next-generation sequencing to isolate mutant genes from forward genetic screens. Nat Rev Genet 2014, 15(10):662-676.

6. Schneeberger $\mathrm{K}$, Ossowski S, Lanz C, Juul T, Petersen AH, Nielsen KL, Jorgensen JE, Weigel D, Andersen SU: SHOREmap: simultaneous mapping and mutation identification by deep sequencing. Nat Methods 2009, 6(8):550-551.

7. Hartwig B, James GV, Konrad K, Schneeberger K, Turck F: Fast isogenic mapping-by-sequencing of ethyl methanesulfonate-induced mutant bulks. Plant Physiol 2012, 160(2):591-600.

8. Austin RS, Vidaurre D, Stamatiou G, Breit R, Provart NJ, Bonetta D, Zhang J, Fung P, Gong Y, Wang PW, McCourt P, Guttman DS: Next-generation mapping of Arabidopsis genes. Plant J 2011, 67(4):715-725.

9. Takagi H, Uemura A, Yaegashi H, Tamiru M, Abe A, Mitsuoka C, Utsushi H, Natsume S, Kanzaki H, Matsumura H, Saitoh H, Yoshida K, Cano LM, Kamoun S, Terauchi R: MutMap-Gap: whole-genome resequencing of mutant F2 progeny bulk combined with de novo assembly of gap regions identifies the rice blast resistance gene Pii. New Phytol 2013, 200(1):276-283.

10. Fekih R, Takagi H, Tamiru M, Abe A, Natsume S, Yaegashi H, Sharma S, Kanzaki H, Matsumura H, Saitoh H, Mitsuoka C, Utsushi H, Uemura A, Kanzaki E, Kosugi S, Yoshida K, Cano L, Kamoun S, Terauchi R: MutMap+: genetic mapping and mutant identification without crossing in rice. PLoS One 2013, 8(7):e68529.

11. Abe A, Kosugi S, Yoshida K, Natsume S, Takagi H, Kanzaki H, Matsumura H, Mitsuoka C, Tamiru M, Innan H, Cano L, Kamoun S, Terauchi R: Genome sequencing reveals agronomically important loci in rice using MutMap. Nat Biotechnol 2012, 30(2):174-178.

12. Minevich G, Park DS, Blankenberg D, Poole RJ, Hobert O: CloudMap: a cloud-based pipeline for analysis of mutant genome sequences. Genetics 2012, 192(4):1249-1269.

13. Monaghan J, Matschi S, Shorinola O, Rovenich H, Matei A, Segonzac C, Gro-Malinovsky F, Rathjen J, MacLean D, Romeis T, Zipfel C: The calcium dependent protein kinase CPK28 buffers plant immunity and regulates BIK1 turnover. Cell Host Microbe 2014, 16:605-615.

14. Sun Y, Li L, Macho AP, Han Z, Hu Z, Zipfel C, Zhou JM, Chai J: Structural basis for flg22-induced activation of the Arabidopsis FLS2-BAK1 immune complex. Science 2013, 342(6158):624-628.

15. Schwessinger B, Roux M, Kadota $Y$, Ntoukakis $V$, Sklenar J, Jones A, Zipfel C: Phosphorylation-dependent differential regulation of plant growth, cell death, and innate immunity by the regulatory receptor-like kinase BAK1. PLoS Genet 2011, 7(4):e1002046.

16. Schulze B, Mentzel T, Jehle AK, Mueller K, Beeler S, Boller T, Felix G, Chinchilla D: Rapid heteromerization and phosphorylation of ligandactivated plant transmembrane receptors and their associated kinase BAK1. J Biol Chem 2010, 285(13):9444-9451.

17. Roux M, Schwessinger B, Albrecht C, Chinchilla D, Jones A, Holton N, Malinovsky FG, Tor M, de Vries S, Zipfel C: The arabidopsis leucine-rich repeat receptor-like kinases BAK1/SERK3 and BKK1/SERK4 are required 
for innate immunity to Hemibiotrophic and Biotrophic pathogens. Plant Cell 2011, 23(6):2440-2455.

18. Heese A, Hann DR, Gimenez-Ibanez S, Jones AM, He K, Li J, Schroeder Jl, Peck SC, Rathjen JP: The receptor-like kinase SERK3/BAK1 is a central regulator of innate immunity in plants. Proc Natl Acad Sci U S A 2007, 104(29):12217-12222.

19. Chinchilla D, Zipfel C, Robatzek S, Kemmerling B, Nurnberger T, Jones JD, Felix G, Boller T: A flagellin-induced complex of the receptor FLS2 and BAK1 initiates plant defence. Nature 2007, 448(7152):497-500.

20. Nekrasov V, Li J, Batoux M, Roux M, Chu ZH, Lacombe S, Rougon A, Bittel P, Kiss-Papp M, Chinchilla D, van Esse HP, Jorde L, Schwessinger B, Nicaise V, Thomma BP, Molina A, Jones JD, Zipfel C: Control of the patternrecognition receptor EFR by an ER protein complex in plant immunity. EMBO J 2009, 28(21):3428-3438.

21. Tedman-Jones JD, Lei R, Jay F, Fabro G, Li X, Reiter WD, Brearley C, Jones JD: Characterization of Arabidopsis mur3 mutations that result in constitutive activation of defence in petioles, but not leaves. Plant J 2008, 56(5):691-703.

22. Zipfel C, Robatzek S, Navarro L, Oakeley EJ, Jones JD, Felix G, Boller T: Bacterial disease resistance in Arabidopsis through flagellin perception. Nature 2004, 428(6984):764-767.

23. Vetter MM, Kronholm I, He F, Haweker H, Reymond M, Bergelson J, Robatzek S, de Meaux J: Flagellin perception varies quantitatively in Arabidopsis thaliana and its relatives. Mol Biol Evol 2012, 29(6):1655-1667.

24. Cingolani P, Platts A, le Wang L, Coon M, Nguyen T, Wang L, Land SJ, Lu X, Ruden DM: A program for annotating and predicting the effects of single nucleotide polymorphisms, SnpEff: SNPs in the genome of Drosophila melanogaster strain w1118; iso-2; iso-3. Fly (Austin) 2012, 6(2):80-92.

25. Huala E, Dickerman AW, Garcia-Hernandez M, Weems D, Reiser L, LaFond F, Hanley D, Kiphart D, Zhuang M, Huang W, Mueller LA, Bhattacharyya D, Bhaya D, Sobral BW, Beavis W, Meinke DW, Town CD, Somerville C, Rhee SY: The Arabidopsis Information Resource (TAIR): a comprehensive database and web-based information retrieval, analysis, and visualization system for a model plant. Nucleic Acids Res 2001, 29(1):102-105.

26. Project IRGS: The map-based sequence of the rice genome. Nature 2005, 436:793-800.

27. Consortium TTG: The tomato genome sequence provides insights into fleshy fruit evolution. Nature 2012, 485:635-641.

28. Schmutz J: Genome sequence of the palaeopolyploid soybean. Nature 2010, 463:178-183.

29. Velasco R, Zharkikh A, Troggio M, Cartwright DA, Cestaro A, Pruss D, Pindo M, FitzGerald LM, Vezzuli S, Reid J, Malacarne G, lliev D, Coppola G, Wardell B, Micheletti D, Macalma T, Facci M, Mitchell JT, Perassolli M, Eldredge G, Gatto P, Oyzerski R, Moretto M, Gutin N, Stefanini M, Chen Y, Segala C, Davenport C, Dematte L, Mraz A, Battilana J, Stormo K, Costa F, Tao Q, Si-Ammour A, Harkins T, Lackey A, Perbost C, Taillon B, Stella A, Solovyev V, Fawcett JA, Sterck L, Vandepoele SK, Fontana P, Gutin A, Ven De Peer Y, Salamini F, Viola R: A high-quality draft concensus sequence of the genome of a heterozygous grapevine variety. PLOS One 2007, 2(12):e1326.

30. Schnable PS: The B73 maize genome: complexity, diversity, and dynamics. Science 2009, 326(5956):1112-1115.

31. Andrews S: FastQC: A Quality Control Tool for High Throughput Sequence Data; 2010. http://www.bioinformatics.babraham.ac.uk/projects/fastqc.

32. Goecks J, Nekrutenko A, Taylor J: Galaxy: a comprehensive approach for supporting accessible, reproducible, and transparent computational research in the life sciences. Genome Biol 2010, 11(8):R86.

33. Giardine B, Riemer C, Hardison RC, Burhans R, Elnitski L, Shah P, Zhang Y, Blankenberg D, Albert I, Taylor J, Miller W, Kent WJ, Nekrutenko A: Galaxy: a platform for interactive large-scale genome analysis. Genome Res 2005, 15(10):1451-1455.

34. Blankenberg D, Von Kuster G, Coraor N, Ananda G, Lazarus R, Mangan M, Nekrutenko A, Taylor J: Galaxy: a web-based genome analysis tool for experimentalists. Curr Protoc Mol Biol 2010, Chapter 19:Unit 1910 11-21.

35. Joshi NA, Fass JN: Sickle: A Sliding-Window, Adaptive, Quality-Based Trimming Tool for FastQ Files (Version 1.21) [Software]; 2011. Available at https://github.com/najoshi/sickle.

36. Li H, Durbin R: Fast and accurate long-read alignment with Burrows-Wheeler transform. Bioinformatics 2010, 26(5):589-595.
37. Li H, Handsaker B, Wysoker A, Fennell T, Ruan J, Homer N, Marth G, Abecasis G, Durbin R: The Sequence Alignment/Map format and SAMtools. Bioinformatics 2009, 25(16):2078-2079.

38. Glazebrook J, Drenkard E, Preuss D, Ausubel FM: Use of cleaved amplified polymorphic sequences (CAPS) as genetic markers in Arabidopsis thaliana. Methods Mol Biol 1998, 82:173-182.

39. Tucker EJ, Huynh BL: Genotyping by high-resolution melting analysis. Methods Mol Biol 2014, 1145:59-66.

40. Fawcelt T: An introduction to ROC analysis. Pattern Recogn Lett 2006, 27(8):861-874

\section{Submit your next manuscript to BioMed Central and take full advantage of:}

- Convenient online submission

- Thorough peer review

- No space constraints or color figure charges

- Immediate publication on acceptance

- Inclusion in PubMed, CAS, Scopus and Google Scholar

- Research which is freely available for redistribution

Submit your manuscript at www.biomedcentral.com/submit
C Biomed Central 